and low back pain of $430(57 \%)$ of women and $303(\% 40,8)$ of men (totally $739(48,9 \%)$ individuals). The point prevalence of low back pain was calculated as 39,9\%(324 individuals), of these 210 were women and 110 were men. The point prevalence was $27,3 \%(\mathrm{n}=21)$ in men aged over 50 and $34,3 \%(\mathrm{n}=93)$ in men aged 50 or under, while it was $57,6 \%(\mathrm{n}=49)$ in women aged over 50 and 42,5\%(n=161) in women aged 50 or under. Conclusion The lifetime rate of neck and low back pain were both higher in women. The increasing age seems to be associated with the point prevalence of low back pain in women contrary to men.

This study is supported by the Akdeniz University Research Fund.

\section{THU0248 FAMILIAL CLUSTERING OF SYSTEMIC LUPUS ERYTHEMATOSUS WITH OTHER AUTOIMMUNE DISEASES}

${ }^{1} R$ Priori, ${ }^{1} F$ Conti, ${ }^{1} E$ Cassarà, ${ }^{2} E$ Medda, ${ }^{2} M A$ Stazi, ${ }^{3} R$ Gerli, ${ }^{4}$ A Manfredi, ${ }^{5} F$ Franceschini, ${ }^{6} \mathrm{MG}$ Danieli, ${ }^{1} \mathrm{G}$ Valesini. ${ }^{1}$ Rheumatology, "La Sapienza" University; ${ }^{2}$ Istituto Superiore Di Sanità, ISS, Roma; ${ }^{3}$ Rheumatic Diseases, University, Perugia; ${ }^{4}$ URIC, H-S. Raffaele, Milano; ${ }^{5}$ Clinical Immunology, University, Brescia; ${ }^{6}$ Internal Medicine, University, Ancona, Italy

\subsection{6/annrheumdis-2001.792}

Background Previous studies have shown the phenomenon of clustering of autoimmune diseases (AD) in families of patients with Systemic Lupus Erythematosus (SLE).

Objectives The purpose of this study was to evaluate the incidence, prevalence and risk to have an $\mathrm{AD}$ in first degree relatives (FDR) of patients with SLE and to investigate if a history of AD in FDR is a risk factor for SLE.

Methods A multicentre case-control study was carried out in Italy. Cases and controls were randomly selected and interviewed using a detailed questionnaire. We conducted stratified analyses according to sex of relatives and/or proband to evaluate whether the relation between SLE in FDR and AD differs by sex. We used the extended generalised estimating equations (EGEE) to measure familial aggregation. The strenght of the association between family history of AD and SLE was measured as an odds ratio (OR). The adjusted OR was obtained by unconditional logistic regression model and the potentially confounding covariates were family size, age and sex of proband.

Results The prevalence of AD among SLE and control FDR was respectively $6.1 \%$ and $1.5 \%(\mathrm{p}<0.01)$. The incidence rate of $\mathrm{AD}$ and control FDR was $1.35 \times 1000$ py and $0.32 \times 1000$ py respectively. The relatives of SLE cases have 4 time probability of having AD compare to relatives of control (RR 4.26; 95\% CI 2.25- 8.03). Using EGEE the adjusted OR taking into account the non-independence of family members was 4.77 (95 CI 2.0711). A risk of 8.57 (95\% CI 3.18-23.1) was detected if the proband is female and $1.40(95 \%$ CI $0.22-8,78)$ if proband is male. The risk of AD is significantly greater for female with a female FDR with SLE (OR 10.36; 95\% CI 2.90- 36.9). No AD was found among SLE spouses, while one case was found among spouses of controls.

Conclusion This case-control study defines the risk to have an autoimmune disorder for SLE first degree relatives. A family history for $\mathrm{AD}$ represents a risk factor for SLE.
THU0249 FAMILY HISTORY OF AUTOIMMUNE DISEASES AS A RISK FACTOR FOR SJÖGREN'S SYNDROME: A CASECONTROL STUDY

${ }^{1} \mathrm{R}$ Priori, ${ }^{1} \mathrm{~F}$ Conti, ${ }^{1} \mathrm{E}$ Cassarà, ${ }^{2} \mathrm{E}$ Medda, ${ }^{2} \mathrm{MA}$ Stazi, ${ }^{3} \mathrm{M}$ Cesarotti, ${ }^{4} \mathrm{P}$ Rovere, ${ }^{5} \mathrm{C}$ Antonioli, ${ }^{6} \mathrm{MG}$ Danieli, ${ }^{7} \mathrm{M}$ Pietrogrande, 'G Valesini. 'Rheumatology, "La Sapienza" University; ${ }^{2}$ Istituto Superiore Di Sanità, ISS, Rome; ${ }^{3}$ Rheumatic Diseases, University, Perugia; ${ }^{4} U R I C, H$ S. Raffaele, Milano; ${ }^{5}$ Clinical Immunology, University, Brescia; ${ }^{6}$ Internal Medicine, University, Ancona, Italy; ${ }^{7} \mathrm{H}-\mathrm{S}$. Paolo, University

10.1136/annrheumdis-2001.793

Background As far as we know, no epidemiological study is available regarding risk factors for Sjögren's syndrome (SS).

Objectives A multicentre case-control study was carried out in Italy to evaluate the relationship between SS and a history of autoimmune diseases (AD) in first degree relatives (FDR).

Methods Cases and controls were randomly selected and interviewed by trained medical doctors using a detailed questionnaire. The strenght of the association between family history of $\mathrm{AD}$ and SS was measured as an odds ratio (OR). The adjusted OR was obtained by unconditional logistic regression model and the potentially confounding covariates were family size, age and sex of proband.

Results A total of 143 eligible SS cases (EEC criteria,1994) and 140 healthy controls were identified. 27/143 (18.8\%) SS and 7/ $140(5 \%)$ controls had one FDR with $\mathrm{AD}, 7 / 143$ (4.9\%) and 1/ $140(0.7 \%)$ had two or more FDR with AD. Considering the proband with one FDR with AD (therefore belonging to a multiplex family) in comparison to probands without any FDR affected (belonging to a simplex family), the adjusted OR was 6.19 (95\% CI 2.3-16.7); the risk is higher if the relatives are two or more: OR 19.2 (95\% CI 1.69-216).

Conclusion This study indicates that a family history of autoimmune disorders is a risk factor for SS. Probands from multiplex families have an higher risk to have SS compared to simplex families. The risk increases with the number of FDR with AD.

\section{THU0250 AUTOIMMUNE DISORDERS IN ICELANDIC MULTICASE SLE FAMILIES}

G Gröndal, K Steinsson. Center for Rheumatology Research and Department of Rheumatology, Landspitalinn, University Hospital, Reykjavik, Iceland

\subsection{6/annrheumdis-2001.794}

\section{Background}

Objectives To evaluate the frequency of autoimmune disorders in Icelandic multicase SLE families.

Methods The study group consisted of 38 SLE patients, 127 first-degree relatives, 37 s-degree relatives and 33 nonconsangineous relatives from multicase SLE families. Pedigree members were evaluated through interviews and physical examination, medical records were reviewed. A standardised protocol for SLE and other autoimmune disorders was used. Serum samples were obtained and autoantibodies measured.

Results Among the SLE patients, 16/38 (42\%) had other autoimmune disorder (s) (11 Sjogrens syndrome/sicca symptoms, 3 interstitial cystitis, 3 psoriasis, 3 pernicious anaemia, 3 thyroid disease, 2 myositis). Twenty six/164 (16\%) first- and seconddegree relatives had various autoimmune disorder (s) (14 Sjogrens syndrome/sicca symptoms, 8 thyroid disease, 6 RA, 3 psoriasis, 3 alopecia, 3 iritis, 1 pernicious anaemia, 1 PBC, 1 MS, 1 DM, 1 AS, 1 reactive athritis, 1 discoid LE). Additionally, 21/ $164(13 \%)$ relatives had autoantibodies. Therefore either 